\title{
Out of the Archive: Faint Features of Bright Galaxies
}

\author{
David Malin \\ Anglo-Australian Observatory, P.O. Box 296, Epping, NSW 2121, \\ Australia \\ Brian Hadley \\ Royal Observatory, Blackford Hill, Edinburgh EHg $3 H J, ~ U K$
}

\begin{abstract}
Simple photographic techniques are used to enhance and combine many images of (mostly) bright galaxies made from plates in the archive of the UK Schmidt Telescope in Edinburgh. The purpose of this project is to reveal extended faint features of these objects that are less than $0.5 \%$ as bright as the night sky. Presented here are the preliminary results of what we intend to be a substantial atlas. The early results suggest that a remarkably large number of apparently normal galaxies reveal unexpected features when very deep images are produced.
\end{abstract}

\section{Introduction}

Several interesting discoveries have been made, often by chance, when applying photographic enhancement techniques to deep plates from the telescopes of the Anglo-Australian Observatory. This paper is a preliminary report on a larger project specifically intended to reveal the faintest features of many nearby, bright or otherwise interesting galaxies. The project involves combining galaxy images from as large a number as possible of deep, uniform UK Schmidt plates of the same region of sky, using photographic amplification and addition techniques. It is hoped to publish the work as an illustrated atlas.

Although the work is not yet complete, it has revealed several interesting new features of well-known galaxies and many low surface brightness abnormalities of apparently 'normal' galaxies. These features often reveal unexpected interactions between pairs and groups of galaxies and surprising distortions in galaxies which seem to be isolated, perhaps reflecting encounters with or anisotropy in otherwise unseen inter-galactic matter. Here we briefly outline the scope of the project and present a few examples from what we hope will be a useful contribution to discussion about what constitutes a 'normal' galaxy.

\section{Method}

Although it has been shown previously that photographic amplification (Malin 1979 ) is a simple but very useful way of revealing very faint, extended objects recorded on 'sky limited' photographic plates, even fainter detail is detectable if 
photographically amplified derivatives from several plates of the same field are combined in register (Malin 1981). This, too, is a relatively simple and rapid process when undertaken photographically, though, of course digital methods can also be used. In both cases, a marked improvement in image quality (signalto-noise ratio) is evident.

In view of the previous successes of the photographic enhancement technique when applied to deep photographic exposures, especially plates from the UK Schmidt telescope at Siding Spring, it was decided to undertake a more systematic survey to exploit the plates from this telescope which are stored in the Plate Library at the Royal Observatory, Edinburgh. This is a collection of over $15000356 \times 356 \mathrm{~mm}$ plates obtained since 1974 in the course of a series of photographic surveys of the southern sky using the Eastman Kodak fine grain IIIa photographic emulsion.

From the beginning, it was intended that the southern sky survey would achieve an unprecedentedly high and uniform standard, and this has resulted in many regions of sky being photographed several times, apart from the nominal pair of sky limited exposures for the major IIIa-J (blue) and IIIa-F (red) surveys. Often this repetition is a consequence of an exposure failing to meet the standard required for an acceptable survey ' $A$ ' grade plate because of any of the numerous practical difficulties that beset astronomical photography. However, these defects may be largely cosmetic, or affect only part of the plate, or reflect poor seeing during the exposure, so there are many deep B grade IIIa-J and IIIa-F plates that are perfectly useful for non-survey projects, especially where the finest image quality is not essential. In addition, there is substantial area of overlap between 6 degree fields photographed on 5 degree centres. There is also a large number of deep plates in the archive which were taken for special, non-survey projects. The result is that there are always at least two plates of all parts of the sky, and sometimes many more for an object that by chance lies in the corner of a field where the overlap region appears on four different plates, or where a succession of attempts has failed to produce an acceptable 'A' grade plate.

\section{Selection of Objects}

One of us (DFM) has for many years maintained a file of 'interesting' objects, mainly galaxies, noted on sky survey plates and copy films. In some cases, photographically amplified, positive film copies of these objects have been made and filed as well. In addition, as part of a photographic survey for dwarf galaxies in the Virgo cluster (Bothun et al. 1987, 1988) a large number of deep plates covering a $10 \times 10$ degree region centred on M 86 were copied with photographic amplification.

More recently, we have undertaken a visual inspection of each of the $900+$ UKST sky survey films of that half of the sky south of the equator in search of galaxies that experience suggested might reveal interesting faint structures. To these were added a number of northern fields that were photographed by the UKST for various projects. Most of these are NGC objects, including many that are bright enough to be in Messier's list, though some are included because they have unusually low surface brightness. The final compilation from all these 
sources consists of about 200 galaxies. The selection was thus on the basis of potential morphological interest rather than on any rigid criteria based on angular size or galaxy type.

Using the UKSCAT program on the computer-readable plate catalogue, it was a fairly simple matter to construct a list of all those UK Schmidt plates where any chosen object appears. Using the same facility, the list could be defined to include only those direct (i.e. non-objective prism) IIIa-J or IIIa-F plates with exposures longer than 30 minutes using a broad-band filter (a sky limited exposure is 60-90 minutes). The list was further refined manually, rejecting ' $\mathrm{C}$ ' or ' $D$ ' grade plates where sufficient ' $A$ ' and ' $B$ ' grades are available. Also, in general, IIIa-Js were preferred to IIIa-Fs because the night sky is significantly darker in the blue pass band. However, in some cases where there were few IIIa-Js, IIIa-Fs were included.

The selected plates of each object were copied on to high contrast film (AgfaGevaert FO 711p) using a diffuse light contact printing device as described by Malin (1978). The contact printer, an Agfa-Gevaert SV400 model, has been modified so the low amplitude variations in sky background density on some plates can be almost entirely compensated by switching off a few of the 25 lamps in the copier, and/or inserting card masks beneath the diffuser in the copier. These large-scale, smoothly varying non-uniformities are of very small amplitude, typically with density differences of 0.1 to 0.3 across $10 \mathrm{~cm}$ of plate, but this simple method of correction produces a copy positive film of remarkably uniform density. This confirms that the original plates, as received from the manufacturer, are uniformly sensitive on the smaller millimetre scales we are interested in. The uniformity of the copy positives greatly simplifies subsequent addition.

Images on each of the positive film copies are projected in precise register and one at a time using the superimposition frame described by Malin (1981). The 'receiving' film is $8 \times 10$ inch Kodak Type 2566 lithographic film which is subsequently developed in a non-lith print developer to obtain high, but not extreme, contrast. The exposure of the several positives is adjusted so that each of the copy positives contributes an equal amount to the sky background density of the subsequent multi-image negative, which is exposed and processed to obtain a sky background density of about 0.8 . This master negative often reveals features that are too faint to be seen by inspection of the individual positives from which it was made. The negative film is contact copied to make a film positive from which negative prints for publication are easily made by contact or enlargement.

\section{Results and Illustrations}

The following four images are typical examples of the kind of faint feature revealed by this process. We have already noted that a surprisingly large fraction of apparently normal galaxies are revealed strange in some way when their luminosity profiles are pushed to a small fraction of the night sky brightness, though is too early as yet to speculate on how general this observation might be. Note that Figs 2-4 are oriented $90^{\circ}$ compared with the usual astronomical convention. 
NGC 5018 (Fig. 1, scale bar $=5$ arcmin) was first recognised as an unusual galaxy by Malin and Carter (1983), who noted shells and a tail and included it in their catalogue of shell galaxies. Its surface photometry has been investigated by Fort et al. (1986), when it was found to have colours typical of an Sb or Sc galaxy, i.e. bluer than for an elliptical galaxy. However, Schweizer (1983) describes it as a true elliptical on the basis of classifications of both de Vaucouleurs and Sandage, but notes that it is dusty. Our deep image strongly suggests that NGC 5018 is interacting with the edge-on disk galaxy NGC 5022, about 6 arcmin to the east. Surprisingly, the disk of NGC 5022 (redshift not known) itself seems hardly disturbed by the encounter, so the pair may be unconnected. Fig. 1 also reveals a distorted and large, very low surface brightness galaxy with an unusually flat luminosity profile, about 5 arcmin N of NGC 5022, which may be part of the same system.

NGC 2708 (Fig 2 and inset, scale bar $=5$ arcmin) was studied by Rubin et al. (1982) as one of a sample of well-characterised Sb galaxies. These authors noted its intense nuclear emission, but there has been no previous reference to the unusual extended faint features. These are rather similar to those revealed in NGC 2698-99, to the north of NGC 2708, and it seems probable that these three galaxies are an interacting trio, similar to the M65-66 group in Leo, especially since the redshifts of NGC 2708 and 2699 are similar.

NGC 1316-17 (Fig. 3, scale bar $=10$ arcmin) is the optical counterpart of the well known southern radio source Fornax A. Our new photograph shows clearly the extensive 'southern loop', and reveals several condensations within it. Revealed here for the first time is a rather fainter, less well defined 'counter loop' to north and northwest of the two main galaxies. The southern loop was first illustrated in the book by Murdin et al. (1979) and is loop L5 in the extensive discussion of these galaxies published by Schweizer (1980). The optical loops do not bear any obvious relationship to the double-lobe structure of radio maps.

NGC 1313 (Fig. 4, scale bar = 10 arcmin) is a nearby, isolated, irregular galaxy in the far southern sky. It appears well resolved on plates from $4 \mathrm{~m}$ class telescopes and shows ample evidence of recent extensive star formation. However, the puzzle is the extent and trigger for the star formation, a problem emphasised by the deep image reproduced here. Despite the lack of any nearby companion, the outer parts of NGC 1313 shows distortions and extensions typical of interacting systems. The picture is further complicated by the very large inverted ' $V$ '-shaped extension to the north, which appears to be attached to the faint structures seen on the eastern side of the galaxy. However, this may be reflection nebulosity associated with the Magellanic Stream through which the galaxy is seen. A detailed optical/radio study of NGC 1313 by Staveley-Smith et al. is in preparation.

\section{References}

Bothun G.D., Impey C.D., Malin D.F. \& Mould J.R.,1987, AJ, 94, 23

Fort B.P., Prieur J.-L., Carter D., Meatheringham S.J. \& Vigroux L., 1986, ApJ, 306,110

Impey C.D., Bothun G.D. \& Malin D.F., 1988, ApJ, 330, 634 
Malin D.F., 1978, Nature, 276, 591

Malin D.F., 1979, Nature, 277, 279

Malin D.F., 1981, J. Phot. Sci., 29, No. 5, 199

Malin D.F. \& Carter D., 1983, ApJ, 274, 534

Murdin P., Allen D.A. \& Malin D., 1979, Catalogue of the Universe, (CUP), p. 41

Rubin V.C., Ford Jr. W. K., Thonnard N. \& Burstein D., 1982, ApJ, 261, 439

Schweizer F., 1980, ApJ, 237, 303

Schweizer F., 1983, in Internal Kinematics and Dynamics of Galaxies, Proc. IAU Symp.100, (Reidel, Dordrecht), p. 319

\section{Discussion}

Hawkins: In order to use your work in a quantitative way, you would need to apply a calibration. How would you go about this?

Malin: It would be difficult but not impossible to derive the sensitometric transfer function from the plate, through the copy positive to the final print. From this, and a value for the average sky brightness for the series of exposures, one could derive a reasonable measure of the surface brightness of any nonsaturated feature in terms of the night sky.

Gilmore: Your photographs are beautiful and a credit to your work. On the basis of this work, can you indicate what fraction of galaxies do not show evidence of abnormality?

Malin: Thanks! I would say that $10-15 \%$ of the very large number of bright galaxies we examined in the course of this and previous work exhibit some anomolous LSB feature. Since many other apparently normal galaxies show peculiar low contrast internal structures, it is becoming increasingly evident that there are few, if any, galaxies that can be described as 'normal'. 

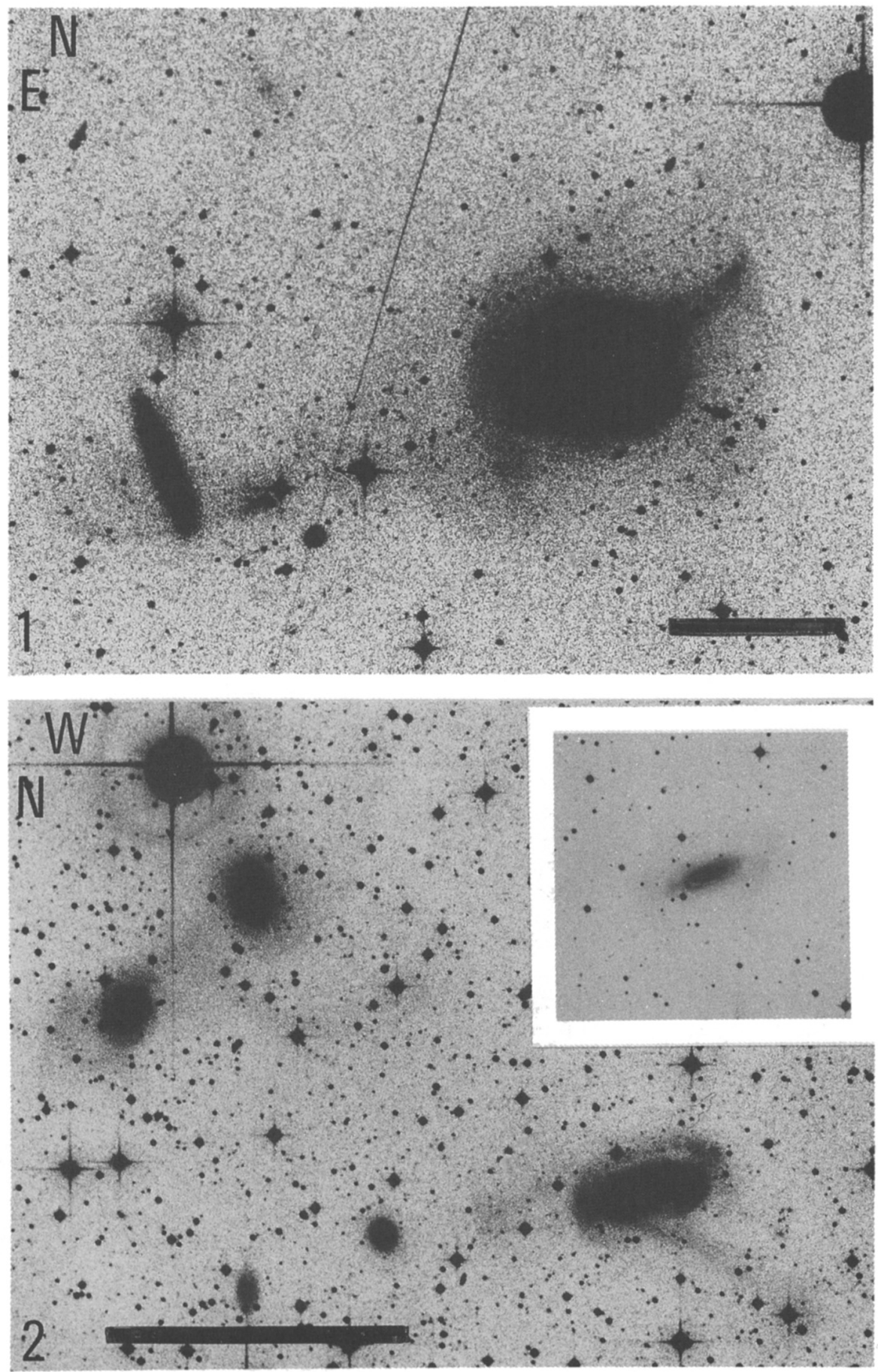

Figures 1 and 2 

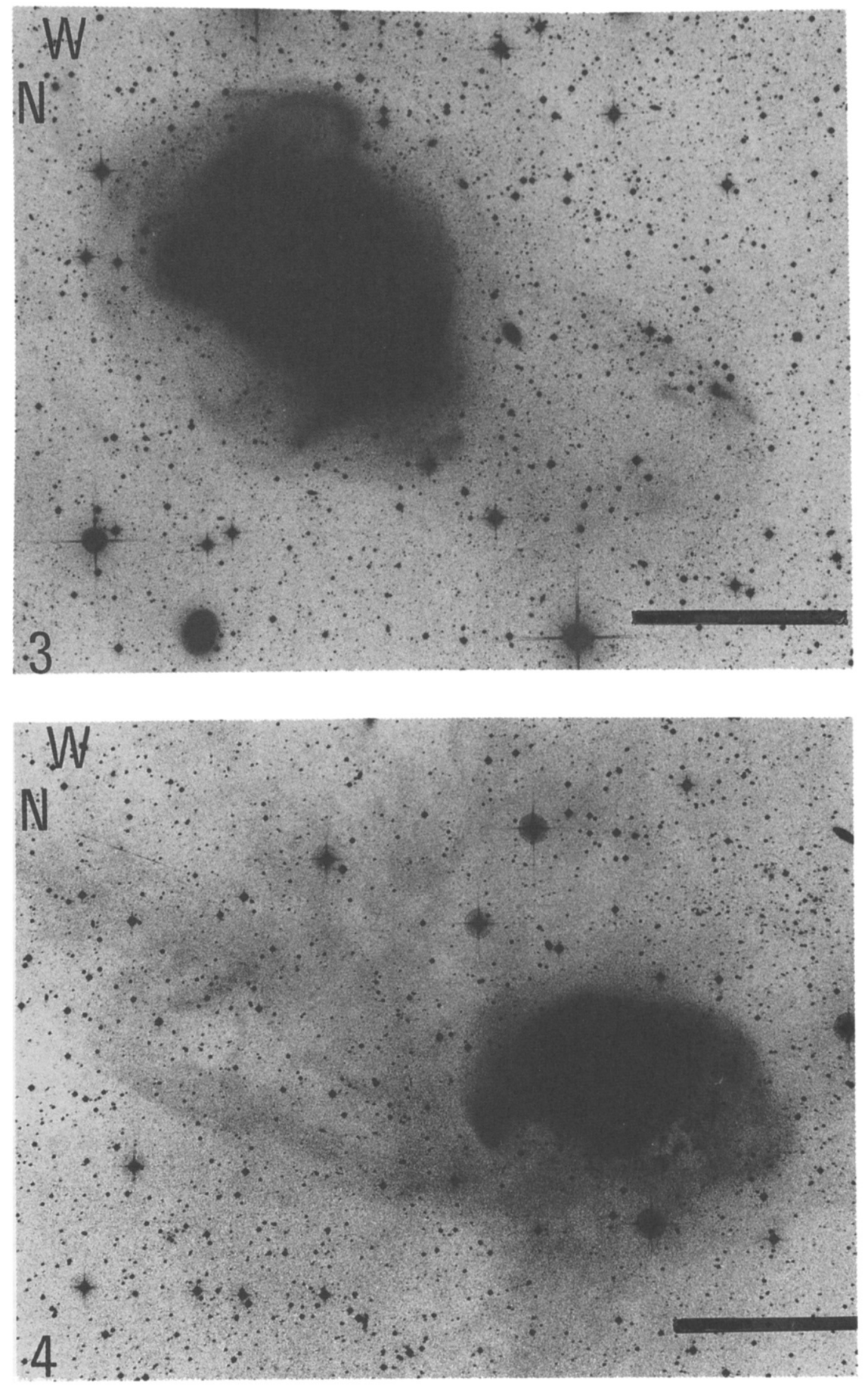

Figures 3 and 4 